\title{
Depinning and dynamics of ac driven vortex lattices in random media
}

\author{
D. Pérez Daroca, * G. S. Lozano, G. Pasquini, and V. Bekeris \\ Departamento de Física, FCEyN, Universidad de Buenos Aires and IFIBA, CONICET, Pabellon 1, Ciudad Universitaria, \\ Buenos Aires, Argentina
}

(Received 6 April 2010; published 19 May 2010)

\begin{abstract}
We study the different dynamical regimes of a vortex lattice driven by ac forces in the presence of random pinning via numerical simulations. The behavior of the different observables is characterized as a function of the applied force amplitude for different frequencies. We discuss the inconveniences of using the mean velocity to identify the depinning transition and we show that instead, the mean quadratic displacement of the lattice is the relevant magnitude to characterize different ac regimes. We discuss how the results depend on the initial configuration and we identify hysteretic effects which are absent in the dc driven systems.
\end{abstract}

DOI: $10.1103 /$ PhysRevB.81.184520

PACS number(s): 74.25.Uv, 74.25.Wx, 74.62.En

\section{INTRODUCTION}

A remarkable diversity of physical systems belong to the category of driven elastic manifolds moving over random landscapes. Extensively studied examples are abundant in the literature: moving vortex lattices in type II superconductors, ${ }^{1-12}$ sliding colloidal particles ${ }^{13,14}$ or charge density wave systems, ${ }^{15}$ magnetic bubble arrays, ${ }^{16}$ driven Wigner crystals, ${ }^{17,18}$ and stripe forming systems. ${ }^{19}$ Among the topics that have recently received much attention is the nature of the depinning mechanisms that occur as the external dc driving force is increased beyond the critical force $F_{C}^{\mathrm{dc}}$, and its relation with proliferation of topological defects in the form of bounded or unbounded disclinations. The dependence of the dynamics with initial conditions has been examined in detail and it was determined that for dc drives, memory of initial conditions is lost at depinning. ${ }^{20}$ A dynamical depinning transition has been identified at a given force $F_{P}^{\mathrm{dc}}$. For larger forces topological defects heal and smectic linear flow is observed. 4,8

It should be noted, however, that most of the research thrust has been specially focused on dc drives. In experiments, an ac field is often applied to order the vortex lattice (VL); the most accepted picture is that the ac field assists the system in an equilibration process, from a disordered metastable configuration to the equilibrium Bragg glass phase, free of dislocations. ${ }^{21}$ However, a large amount of results, in both experiments ${ }^{22,23}$ and simulations, ${ }^{13,24-27}$ show that, in some cases, an ac drive can disorder the VL, and that oscillatory dynamics plays an essential role.

The oscillatory dynamics of the VL is in itself a broad field that is not completely understood. The main porpoise of this work is to provide a more comprehensive description, using numerical simulations, of ac driven vortex lattices over a random distribution of pinning sites. We explore the effect of the applied force amplitude for different frequencies, starting from different initial configurations. The VL mean velocity, which can be dephased from the excitation, is no longer an adequate observable to detect depinning and we show that instead, the mean quadratic displacement of the lattice is the relevant magnitude to identify depinning and dynamical ac regimes. The depinning transition becomes a crossover as is described below. The pinned linear Campbell regime, char- acterized by a linear, frequency independent response where losses are negligible, ${ }^{28}$ is also identified and simulations are compared with analytical calculations.

The paper is organized as follows. In Sec. II, we describe in detail the numerical simulations; starting with the model (Sec. II A), following with the numerical procedures (Sec. II B), and giving the definition of the observables (Sec. II C). In Sec. III, we present and discuss the results, and in Sec. IV, we highlight the main conclusions.

\section{NUMERICAL SIMULATIONS}

\section{A. Model}

In our simulations, we consider $N_{v}$ rigid vortices with coordinates $\mathbf{r}_{i}$ in a two-dimensional rectangle of size $L_{x}$ $\times L_{y}$ that evolve according to the dynamics

$$
\mathbf{F}_{i}-\eta \mathbf{v}_{i}=0,
$$

where $\mathbf{v}_{i}$ its velocity and $\eta$ the Bardeen-Stephen viscosity coefficient and $\mathbf{F}_{i}$ is given by the sum of the vortex-vortex interaction, the pinning attraction, and the Lorentz force

$$
\mathbf{F}_{i}=\mathbf{F}_{i}^{v v}+\mathbf{F}_{i}^{v p}+\mathbf{F}_{i}^{L}
$$

The vortex-vortex interaction per unit length is given by

$$
\mathbf{F}_{i}^{v v}=\sum_{j \neq i}^{N_{v}} \mathbf{F}^{v v}\left(\mathbf{r}_{i}-\mathbf{r}_{j}\right)
$$

where

$$
\mathbf{F}^{v v}\left(\mathbf{r}_{i}-\mathbf{r}_{j}\right)=\frac{\phi_{0}^{2}}{8 \pi^{2} \lambda^{3}} f_{v v} K_{1}\left(\frac{\left|\mathbf{r}_{i}-\mathbf{r}_{j}\right|}{\lambda}\right) \hat{\mathbf{r}}_{i j} .
$$

Here, $\phi_{0}$ is the quantum of magnetic flux, $\lambda$ is the London penetration length, and $K_{1}$ is the modified Bessel function. The parameter $f_{v v}$ is dimensionless and can be related to the stiffness of the vortex lattice (see Ref. 24). The $N_{p}$ pinning centers are supposed to be located at random positions $\mathbf{R}_{j}$, and their interaction with vortices is modeled by

$$
\mathbf{F}^{v p}\left(\mathbf{r}_{i}\right)=\sum_{j=1}^{N_{p}}=\mathbf{F}^{v p}\left(\mathbf{r}_{i}-\mathbf{R}_{j}\right),
$$




$$
\mathbf{F}^{v p}\left(\mathbf{r}_{i}-\mathbf{R}_{j}\right)=-F_{j}^{p} e^{-\left(\left|r_{i}-R_{j}\right| / r_{p}\right)^{2}}\left(\mathbf{r}_{i}-\mathbf{R}_{j}\right),
$$

here, $F_{j}^{p}$ (chosen from a Gaussian distribution with mean value $F^{p}=0.2$ and a standard deviation of $\left.0.1 F^{p}\right)$ and $r_{p}$ tune the strength and range of the interaction.

The Lorentz force per unit length is given by $F_{L}=\phi_{0} J_{\text {ext }}$ $\times z$ where $J_{\text {ext }}$ is the external driving current density and $z$ is the versor perpendicular to the plane.

Following Ref. 24, we measure lengths in units of $\lambda$, forces (per unit length) in units of $f_{0}=\frac{\phi_{0}^{2}}{8 \pi^{2} \lambda^{3}}$, time in units of $t_{0}=\eta \lambda / f_{0}$, and frequency in units of $\omega_{0}=1 / t_{0}$. In our simulation, we will consider $N_{v}=1600, L_{x}=40 \lambda, L_{y}=\sqrt{3} L_{x} / 2, N_{p}$ $=25 N_{v}$, and $r_{p}=0.2 \lambda$.

Concerning the numerical details, the equations of motion are integrated using a standard Euler algorithm with step $h$ $=0.04 t_{0}$, and a hard cutoff $\Lambda=4 \lambda$ in calculating the vortexvortex force. When calculating physical observables we average over four realizations of disorder.

\section{B. Procedures}

The ac drive is simulated with an external square ac force of the form

$$
F_{L}^{\mathrm{ac}}= \begin{cases}f_{L} & \text { if } n T<t<n T+\frac{T}{2} \\ -f_{L} & \text { if } n T+\frac{T}{2}<t<(n+1) T .\end{cases}
$$

We study vortex lattice evolution as we vary the force in a slow ramping of $f_{L}$ starting from different initial conditions. In each case, we leave the system evolving from the initial configuration with zero external force for 5 cycles. This free evolution creates or annihilates a low density of defects and becomes our metastable initial state. A numerical realization will be obtained by ramping from $f_{L}$ to $f_{L}+\Delta f_{L}$, applying $N_{a}$ cycles this new force, and then allowing the system to relax for $N_{w}$ cycles at zero force, before reassuming the ramping. Measuring is performed during the last cycle, just before switching off the applied force (i.e., during cycle $N_{a}$ ).

\section{Observables}

It is by now well known that the mobility of the VL is affected by the topology of the VL configuration. A common observable used to characterize the VL configuration is the density of lattice defects, $n_{d}$, (i.e., vortices with five or seven neighbors in the Delaunay triangulation).

The mobility itself is characterized by the vortex velocity. The instantaneous mean vortex velocity in the direction of the applied force at time $t$ is

$$
v(t)=\frac{1}{N_{v}} \sum_{i}^{N_{v}} v_{i}(t) .
$$

In the dc case, the $v$ vs $F_{L}$ curves can be directly related with experimental current-voltage characteristics ( $V-I$ curves). In the ac case the relationship between velocity and force and the experimental $V$ - $I$ curve is more subtle, as the phase between the applied force and the velocity plays an essential role.

We then define the half cycle mean velocity in the $n$th cycle as

$$
v_{n}=\frac{2}{T} \int_{n T}^{n T+T / 2} v(t) d t
$$

Notice that the half cycle velocity takes into account this phase factor. We estimate the mean phase factor $\varphi_{n}$ by applying a sinusoidal $F_{L}(t)$ and calculating the phase between $F_{L}$ and the first harmonic of the mean velocity $v(t)$ in the $n$th cycle.

Moreover, a quantity of interest is the average quadratic displacement defined as

$$
\left\langle\delta X^{2}\right\rangle\left(t_{2}, t_{1}\right)=\frac{1}{N_{v}} \sum_{i=1}^{N_{v}}\left[x_{i}\left(t_{2}\right)-x_{i}\left(t_{1}\right)\right]^{2} .
$$

In the ac case, we can take $t_{2}=t_{1}+p T$, the mean quadratic displacement in the force direction after performing $p$ oscillations. This quantity, as we will show below, is very useful for describing ac drives.

\section{RESULTS AND DISCUSSION}

Throughout the work we have explored the behavior of the system as a function of the ac amplitude. Recent numerical studies on a similar system (colloids under the influence of an external ac drive and quenched disorder) ${ }^{13}$ have found a crossover from a low drive-highly disordered phase to a high-drive-low-disordered phase. In all cases considered in Ref. 13 the initial configuration was highly disordered. Our results confirm the existence of the high-drive-lowdisordered phase but we will show that when more general initial conditions are considered then richer, low drive phases emerge.

Results starting from an ordered configuration are summarized in Fig. 1 and Fig. 2. In all the cases $N_{a}=6$ cycles have been applied with each $f_{L}$ force. We have checked our results for larger values of $N_{a}$ (up to $N_{a}=60$ ) and we have not found any qualitative difference. The observables $v_{n}$ [Eq. (9)] and $n_{d}$ have been calculated in the $n$th cycle before switching off $f_{L}$. In Fig. 1, we show the behavior of the defect density $n_{d}$ [Fig. $\left.1(\mathrm{a})\right]$ and the characteristic $v_{n}-f_{L}$ curve [Fig. 1(b)] for a particular choice of vortex-vortex interaction $\left(f_{v v}=0.8 f_{0}\right)$, at various frequencies $\omega$. In the dc limit (shown in red full circles) we clearly identify three different regimes, a pinned lattice for $f_{L}<F_{c}^{\mathrm{dc}}$, a disordered flow (plastic) region for $F_{c}^{\mathrm{dc}}<f_{L}<F_{p}^{\mathrm{dc}}$ and a flowing (smectic) linear regime for $f_{L}>F_{p}^{\mathrm{dc}}$ consistent with previous work. ${ }^{20}$ While the existence of $F_{p}^{\text {ac }}$ can be inferred form these ac curves, as the value for which all curves that present plastic motion merge, the distinction between the pinned and plastic regimes is less apparent. This is so, because even a weak driving force produces oscillations of vortices around their pinning centers with finite mean velocity. This is a strong argument to abandon the mean velocity as the ad- 


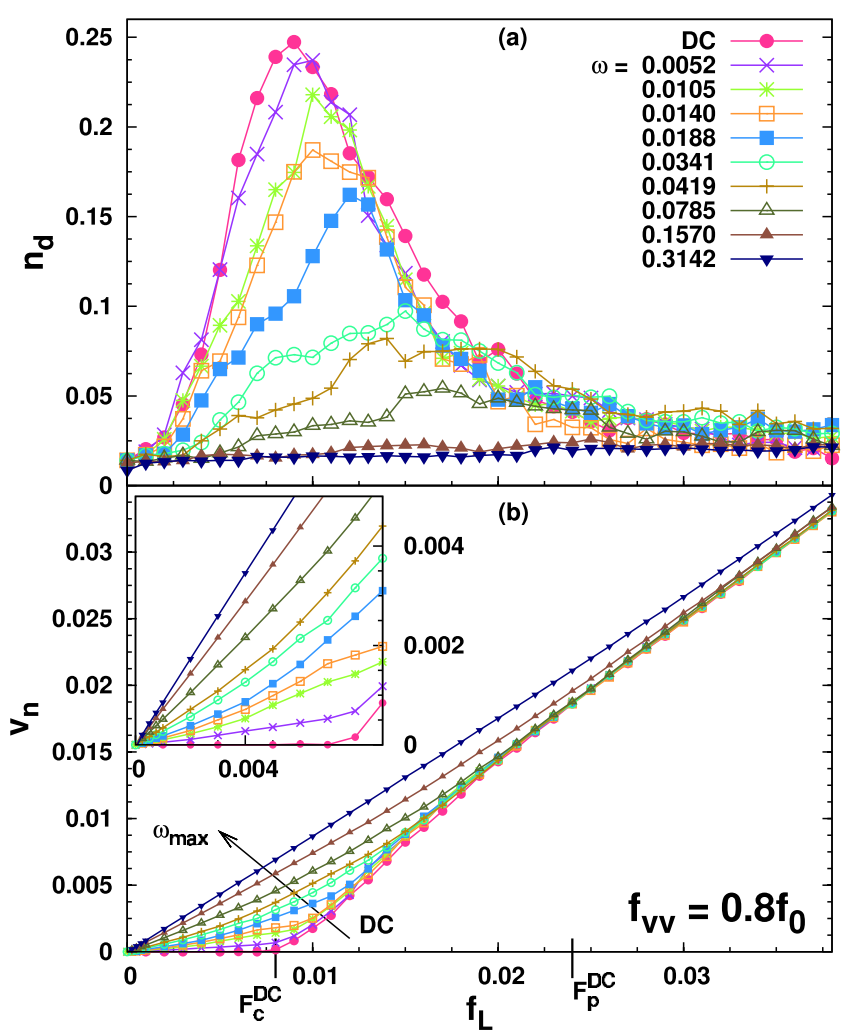

FIG. 1. (Color online) (a) Number of topological defects $n_{d}$ and (b) half-cycle mean velocity $v_{n}$ (see text) as a function of the amplitude of the applied Lorentz force $f_{L}$ for different frequencies $\omega$. All the curves are calculated slowly ramping $f_{L}$, from an ordered VL configuration. Inset: zoom of $v_{n}\left(f_{L}\right)$ curves at low amplitudes: $v_{n}>0$ at finite frequency.

equate observable to indicate depinning. The inset of Fig. 1(b) shows an enlarged area for small driving forces: at very small ac amplitudes a linear Campbell regime holds but the response becomes nonlinear at amplitudes well bellow $F_{c}^{\mathrm{dc}}$. The definition of a critical force in the ac response is therefore not so obvious, and we will discuss this point in detail later.

In the ac curves, we distinguish two frequency regimes: at low frequencies $\left(\omega \lesssim 0.02 \omega_{0}\right.$ in our simulation), restoring and pinning forces prevail over losses, whereas at highfrequencies viscous drag dominates. The low-frequency regime is characterized by a highly nonlinear response at intermediate ac amplitudes, associated with a pronounced peak in $n_{d}$. This feature smears at higher frequencies, the density of topological defects decreases, and the nonlinearity is less apparent, going to a linear "ohmic" regime (blue down triangles) at very high-frequency $\left(\omega \gtrsim 0.2 \omega_{0}\right)$ where dissipative forces govern. Memory and history effects occur when pinning and elastic forces compete and both prevail over viscous forces. Therefore, in the following analysis we will focus in the low-frequency regime.

In Fig. 2, we plot together various observables as a function of the AC amplitude for a fixed frequency $\omega$ $=0.0188 \omega_{0}$, in a procedure analogous to that explained in the description of Fig. 1. Figure 2(a) displays the modulus of the half cycle mean velocity $v_{n}$ (left axis) and the estimated

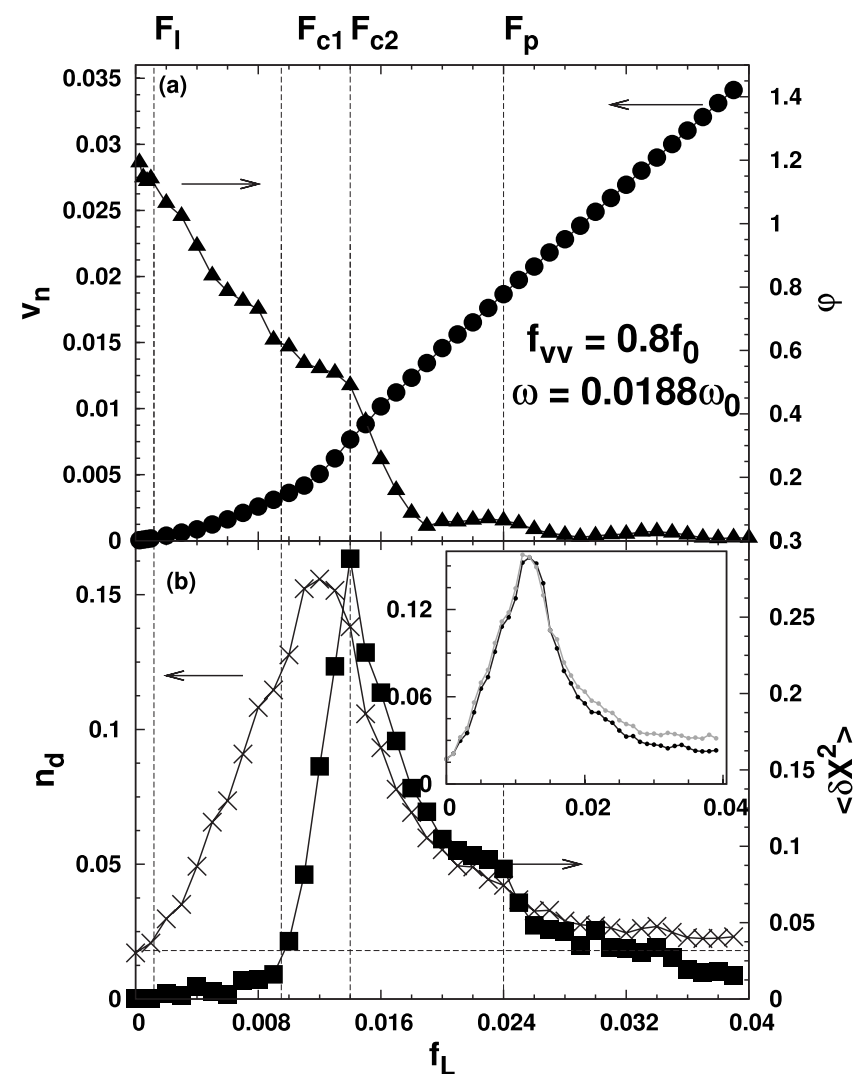

FIG. 2. (a) (left) $v_{n}$ phase factor (a) (right) $\varphi$, (b) (left) $n_{d}$ and mean quadratic displacement (b) (right) $\left\langle\delta X^{2}\right\rangle$ as a function of $f_{L}$ at $\omega=0.0188 \omega_{0}$. Vertical dashed lines indicate the different ac regions discussed in the text. The depinning of the VL occurs in a crossover region between $F_{c 1}$ and $F_{c 2}$, where $\left\langle\delta X^{2}\right\rangle$ grows. Inset: comparison between $n_{d}$ before (black full circles) and after (gray full circles) $N_{w}$ cycles without applied force (see text). Beyond the depinning region the moving lattice configurations are unstable and relax.

phase factor $\varphi_{n}$ (right axis). In Fig. 2(b) the density of defects $n_{d}$ (left axis) and the mean quadratic displacement $\left\langle\delta X^{2}\right\rangle$ after 5 cycles (right axis) are shown. The dashed horizontal line in Fig. 2(b) indicates de density of defects $n_{d r}$ that are spontaneously created (without any applied force), and correspond to the initial more ordered configuration. A still more ordered configuration is unstable.

We identify various regimes. At very low-ac amplitudes $\left(f_{L}<F_{l}\right)$ a linear $v_{n}\left(f_{L}\right)$ holds. The velocity is mainly out of phase. The VL configuration remains unchanged, because vortices are trapped and can only perform small harmonic oscillations around their initial positions. This motion is reversible, and therefore $\left\langle\delta X^{2}\right\rangle=0$. In the other limit, at very high-ac amplitudes $\left(f_{L}>F_{p}\right)$, there is a dynamic reordering and the pinning potential is completely smeared. There is a linear in phase Ohmic response.

In the intermediate range $F_{l}<f_{L}<F_{p}$, the response is nonlinear. A very rich behavior with different nonlinear regimes may be observed. In the first nonlinear region, at small amplitudes $\left(F_{l}<f_{L}<F_{c 1}\right)$, the velocity is small, and there is not an appreciable displacement in the direction of the force (i.e., $\left\langle\delta X^{2}\right\rangle \sim 0$ ); plastic random displacement produces a huge number of dislocations. However, most of the vortices 
remain trapped around the pinning sites. Depinning occurs in a crossover region, between $F_{c 1}$ and $F_{c 2}$. We identify the beginning of the depinning region at the force $F_{c 1}$, where vortices move in average distances larger than the pinning radius $r_{p}$. In this region, the irreversible displacement grows with $f_{L}$ and is reflected in the growth of $\left\langle\delta X^{2}\right\rangle$ that reaches its maximum at the second force $F_{c 2}$; as this happens, the density of defects attains its maximum and the slope of $v_{n}\left(f_{L}\right)$ grows. Above $F_{c 2}$, in the upper nonlinear region $\left(F_{c 2}<f_{L}\right.$ $<F_{p}$ ), the mean phase factor drastically decreases (i.e., dissipation becomes relevant), while the motion becomes more reversible and the VL more ordered. This is accompanied by a smooth decrease in the $v_{n}\left(f_{L}\right)$ slope that approaches the final linear relationship. Another interesting point to remark is that VL configurations that have similar density of defects at both sides of the depinning transition are qualitatively different. This difference can be detected, observing the relaxation of the VL configurations after removing the applied force. In the inset of Fig. 2(b), we compare the density of defects $n_{d}$ before switching off the applied force (black full circles) with the same observable, $N_{w}$ cycles after removing the applied force (gray full circles). While for forces lower than $F_{c 2}$, the vortices move around pinning sites in robust metastable configurations, beyond the depinning transition the moving lattice configurations are unstable and relax toward a state with more defects.

We now turn to analyze the various ac regimes starting from different initial conditions. In Fig. 3 the density of defects $n_{d}$ [Fig. 3(a)] and the mean quadratic displacement $\left\langle\delta X^{2}\right\rangle[$ Fig. 3(b)] as a function of the ac force are shown for different initial conditions and ac histories. The results described in the previous paragraph (starting from an ordered $\mathrm{VL}$ and increasing the ac force) are plotted in red crosses. Analogous results obtained with a similar ac protocol but starting from the more disordered metastable configuration are plotted in blue dots, and results with an initial intermediate $n_{d}$ are plotted in green triangles. As in the dc case studied in Ref. 20, the different $n_{d}$ curves merge for $f_{L}$ larger than a threshold value. For the dc case, this threshold value was identified with $I_{c}^{\mathrm{dc}}$, the critical current at depinning. As we mentioned before, the identification of the critical current $I_{c}^{\mathrm{ac}}$ is less obvious in the ac case, and we notice that the $n_{d}\left(f_{L}\right)$ curves merge around $F_{c 2}$. In magenta squares, we show the behavior of $n_{d}$ and $\left\langle\delta X^{2}\right\rangle$ for decreasing (open squares) and a subsequent increasing (full squares) external driving. We observe the presence of hysteresis in the region between $F_{c 1}$ and $F_{p}$. Consistently, for increasing forces, both the density of defects and the average quadratic displacement result smaller than for increasing forces. Below $F_{c 1}$ pinning forces dominate, and above $F_{p}$ they are completely smeared. In the intermediate region, the strong competition gives rise to hysteresis. This hysteretic effect beyond the critical force has not been observed in the $\mathrm{dc} \operatorname{case}^{20}$ and it seems to be a new genuine effect of the ac case.

As another striking result, the behavior of $\left\langle\delta X^{2}\right\rangle$ vs $F_{L}$ is independent of the initial condition for the whole driving force range. The reorganization of vortex defects by ac drives in initially different configurations, and for different ac protocols, surprisingly does not involve different quadratic displacements. This observable is univocally related

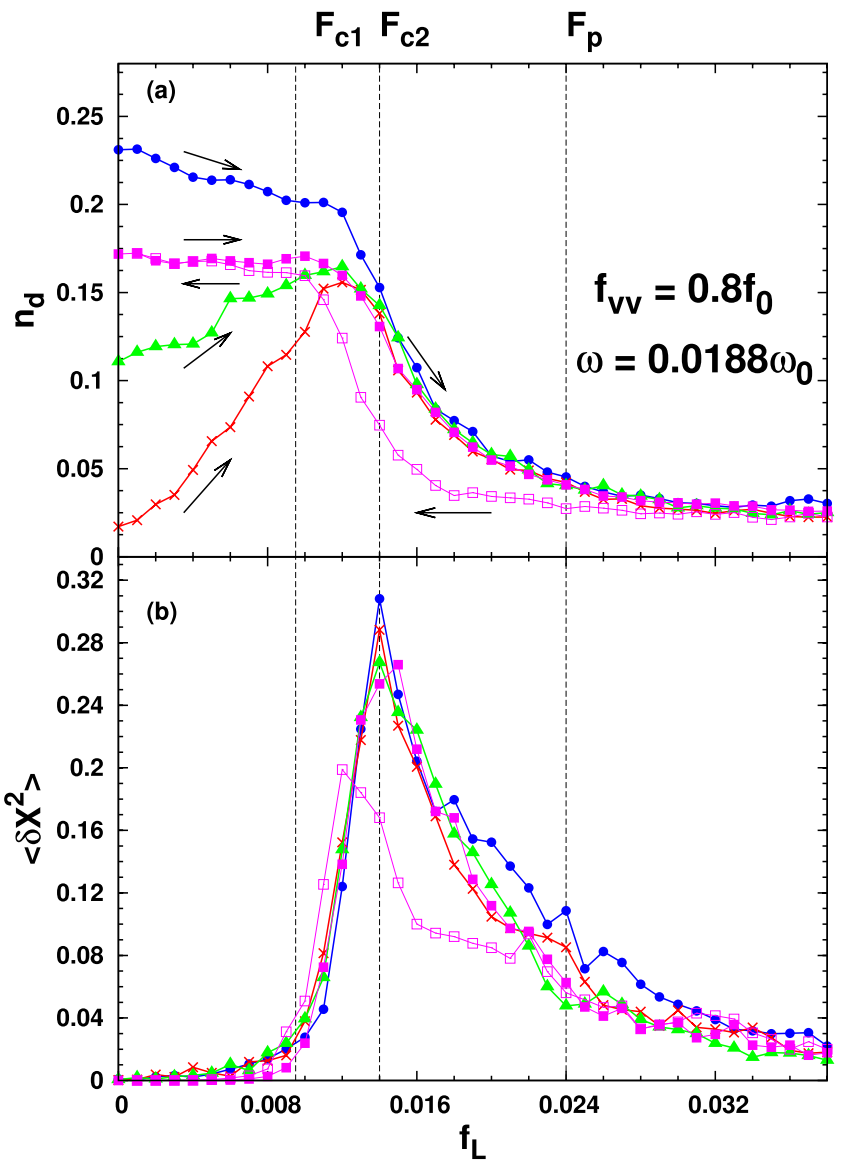

FIG. 3. (Color online) (a) $n_{d}$ as a function of $f_{L}$ starting from different initial configurations. Arrows indicate the direction of change of $f_{L}$. Above $F_{c 2}$ all the $n_{d}\left(f_{L}\right)$ curves taken with increasing $f_{L}$ merge. Hysteresis is observed (full and empty magenta squares). (b) $\left\langle\delta X^{2}\right\rangle\left(f_{L}\right)$ curves corresponding to the same processes described in (a). This observable is independent of the initial condition for the whole driving force range. Hysteresis is observed (full and empty magenta squares).

with the applied force and seems to be the best one to characterize the ac regimes.

Finally, we discuss in more detail the linear regime obtained at very small ac driving forces. In the harmonic approximation we can write the mean restoring force [averaging $\mathbf{F}_{i}^{v v}+\mathbf{F}_{i}^{v p}$ in Eq. (2)] as $\mathbf{F}_{\text {rest }}=-\alpha x$, where $\mathbf{x}$ denotes the average displacement of vortices from their equilibrium position and $\alpha$ is the Labusch parameter. ${ }^{28}$ Within this approximation, Eq. (1) can be written as

$$
\eta \dot{x}+\alpha x=F_{L}^{\mathrm{ac}} .
$$

If we consider a semicycle for which $F_{L}^{\text {ac }}$ is positive, the solution of Eq. (11) is

$$
x(t)=f_{L} / \alpha+\left[x_{0}\left(t_{n}\right)-f_{L} / \alpha\right] e^{-\alpha / \eta\left(t-t_{n}\right)},
$$

where $n T<t<n t+\frac{T}{2}$.

This equation provides a recurrence relation for $x_{0}\left(t_{n}\right)$ that can be solved explicitly. In the limit $n \rightarrow \infty$ (the stationary regime) this gives 


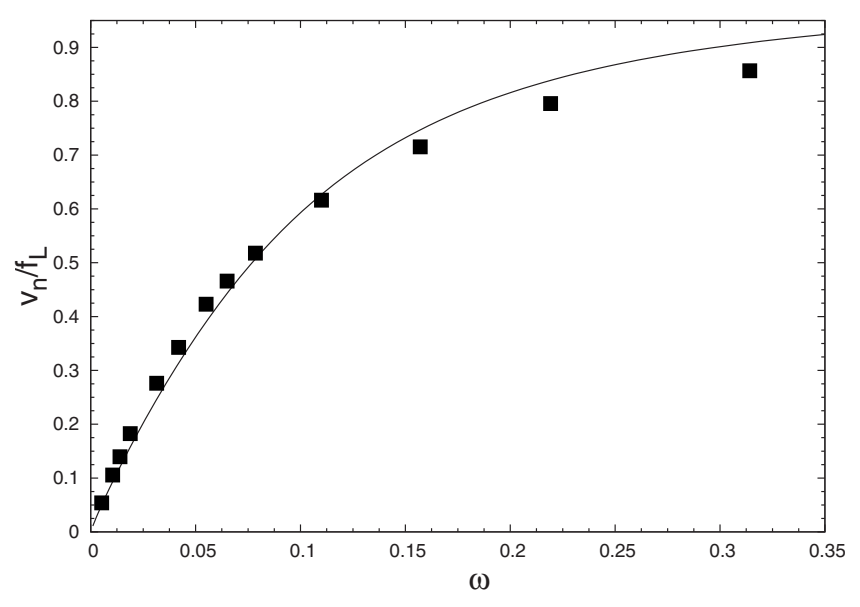

FIG. 4. Normalized half cycle mean velocity $v_{n} / f_{L}$ as a function of frequency $\omega$ in the linear regime, at low $F_{L}$. Fit with Eq. (14) (continuous line), considering a Gaussian distribution for the Labusch constant, gives $\frac{\alpha_{L}}{\eta} \sim \omega_{c}=0.11 \pm 0.05$.

$$
x_{0}\left(t_{n}\right)=-\frac{f_{L}}{\alpha} \tanh \left(\frac{\alpha T}{4 \eta}\right)
$$

and the average velocity over half-cycle as

$$
\overline{v_{n}}=\frac{2 \omega f_{L}}{\alpha \pi} \tanh \left(\frac{\alpha \pi}{2 \eta \omega}\right) .
$$

On the other hand, numerically we have performed different calculations of the half cycle mean velocity in the $n$th cycle $v_{n}$ [according to Eq. (9)] as a function of $\omega$ for different values of $f_{L}$, so we can average over $f_{L}$ and study the behavior of $\overline{v_{n}} / f_{L}$. In addition, as we did before, we have performed averages over different realizations of pinning centers. This fact implies that indeed $\alpha$ is a random variable that as a first approximation can be assumed to be Gaussian distributed and characterized by its mean value $\bar{\alpha}$ and standard deviation $\sigma_{\alpha}$.

In Fig. 4, we show the results of the simulation, where we have used seven values of $f_{L}$ from $0.00025 f_{0}$ to $0.004 f_{0}$ and four realizations over disorder. Our fit gives $\bar{\alpha}=0.11$ and $\sigma_{\alpha}=0.05$.

A critical frequency may be estimated as $\omega_{c} \sim \frac{\bar{\alpha}}{\eta}$ $=0.11 \pm 0.05$. We are able now to justify the two frequency regimes introduced at the beginning of this section.

\section{CONCLUSIONS}

We have presented a comprehensive description of the dynamics of ac driven vortex lattices over a random distribution of pinning sites, focusing the study in the low frequency regime, $\omega \ll \omega_{c}$, where pinning and elastic forces prevail over viscous forces. This critical frequency $\omega_{c}$ has been estimated by fitting results of numerical simulations at low-ac amplitudes with that predicted by an analytical model.
One of the most important results is the behavior of the mean quadratic displacement $\left\langle\delta X^{2}\right\rangle\left(f_{L}\right)$; strikingly it is independent of the initial condition for the whole driving force range. This observable is univocally related with the applied force, and seems to be the best one to characterize the ac regimes. This should be contrasted with the mobility that can be large even in pinned ac driven lattices.

As expected, a linear Campbell regime holds at very low-ac amplitudes $\left(f_{L}<F_{l}\right)$ whereas the response is Ohmic at very high-ac amplitudes $\left(f_{L}>F_{p}\right)$ where there is a dynamic reordering and the pinning potential is completely smeared. In both linear responses the motion is reversible and $\left\langle\delta X^{2}\right\rangle=0$. In all the intermediate range $F_{l}<f_{L}<F_{p}$, the response is nonlinear and a very rich behavior with different nonlinear regimes may be observed.

The second important issue of this work is that depinning occurs in a crossover region $\left(F_{c 1}<f_{L}<F_{c 2}\right)$ where $\left\langle\delta X^{2}\right\rangle$ grows with $f_{L}$ and vortices move in average distances much larger than the pinning radius. In the first nonlinear region, below the depinning transition $\left(F_{l}<f_{L}<F_{c 1}\right)$ there is not an appreciable displacement in the direction of the force (i.e., $\left.\left\langle\delta X^{2}\right\rangle \sim 0\right)$; plastic random displacement produces a huge number of dislocations but most of the vortices remain trapped around the pinning sites. The density of defects $n_{d}$ is strongly dependent on the initial configuration.

Beyond the depinning region the memory of the initial configuration is lost. Dynamics becomes more and more reversible as $\left\langle\delta X^{2}\right\rangle$ decreases. Dissipation becomes relevant and the VL reorders. The moving vortex lattice configurations become unstable and relax after switching off the applied force. However, there is a large region $\left(F_{c 2}<f_{L}<F_{p}\right)$ where plastic motions are still present, as can be inferred from $\left\langle\delta X^{2}\right\rangle>0$ and the nonlinear response. Below the plastic threshold $F_{p}$, an hysteresis in the applied force, absent in dc driven lattices, is observed, showing once again the rich particularities of oscillatory dynamics.

Most of the papers dealing with molecular dynamics simulations that have inspired this work, are straightforwardly related to dc transport experiments. Applied forcevelocity in calculations matches applied current-voltage in dc experiments. In our simulation we control explicitly the applied force and we calculate observables as velocity. An interesting question is how these results could be interpreted in terms of ac experiments in which an external oscillating magnetic field is the control parameter like in rf surface impedance measurements, or in ac susceptibility measurements. The relationship is not straightforward because the ac field and the Lorentz force are not trivially related and, besides, the ac penetration length has to be taken into account. ${ }^{29-31}$ This will be the issue of a coming work.

\section{ACKNOWLEDGMENTS}

This work was partially supported by CONICET under Grants No. PIP 1212/09 and No. PIP 112200801 00930, and UBACyT under Grants No. X13, No. X123, and No. X166. 
*daroca@df.uba.ar

${ }^{1}$ H. J. Jensen, A. Brass, and A. J. Berlinsky, Phys. Rev. Lett. 60, 1676 (1988).

${ }^{2}$ S. Bhattacharya and M. J. Higgins, Phys. Rev. Lett. 70, 2617 (1993).

${ }^{3}$ S. Ryu, M. Hellerqvist, S. Doniach, A. Kapitulnik, and D. Stroud, Phys. Rev. Lett. 77, 5114 (1996).

${ }^{4}$ A. E. Koshelev and V. M. Vinokur, Phys. Rev. Lett. 73, 3580 (1994).

${ }^{5}$ K. Moon, R. T. Scalettar, and G. T. Zimányi, Phys. Rev. Lett. 77, 2778 (1996).

${ }^{6}$ M. C. Faleski, M. C. Marchetti, and A. A. Middleton, Phys. Rev. B 54, 12427 (1996).

${ }^{7}$ C. J. Olson, C. Reichhardt, and F. Nori, Phys. Rev. Lett. 81, 3757 (1998).

${ }^{8}$ P. Le Doussal and T. Giamarchi, Phys. Rev. B 57, 11356 (1998).

${ }^{9}$ H. Fangohr, S. J. Cox, and P. A. J. de Groot, Phys. Rev. B 64, 064505 (2001).

${ }^{10}$ C. J. Olson, C. Reichhardt, and F. Nori, Phys. Rev. Lett. 80, 2197 (1998).

${ }^{11}$ L. Balents, M. C. Marchetti, and L. Radzihovsky, Phys. Rev. B 57, 7705 (1998).

${ }^{12}$ A. B. Kolton, D. Domínguez, and N. Gronbech-Jensen, Phys. Rev. Lett. 83, 3061 (1999).

${ }^{13}$ C. Reichhardt and C. J. Olson Reichhardt, Phys. Rev. E 73, 046122 (2006).

${ }^{14}$ C. Reichhardt and C. J. Olson, Phys. Rev. Lett. 89, 078301 (2002).

${ }^{15}$ L. Balents and M. P. A. Fisher, Phys. Rev. Lett. 75, 4270 (1995).
${ }^{16}$ R. Seshadri and R. M. Westervelt, Phys. Rev. Lett. 70, 234 (1993).

${ }^{17}$ M. C. Cha and H. A. Fertig, Phys. Rev. B 50, 14368 (1994).

${ }^{18}$ C. Reichhardt and C. J. Olson Reichhardt, Phys. Rev. Lett. 93, 176405 (2004).

${ }^{19}$ C. Reichhardt, C. J. Olson Reichhardt, I. Martin, and A. R. Bishop, Phys. Rev. Lett. 90, 026401 (2003).

${ }^{20}$ M. Chandran, R. T. Scalettar, and G. T. Zimányi, Phys. Rev. Lett. 89, 187001 (2002).

${ }^{21}$ H. Beidenkopf, N. Avraham, Y. Myasoedov, H. Shtrikman, E. Zeldov, B. Rosenstein, E. H. Brandt, and T. Tamegai, Phys. Rev. Lett. 95, 257004 (2005).

${ }^{22}$ G. Pasquini, D. P. Daroca, C. Chiliotte, G. S. Lozano, and V. Bekeris, Phys. Rev. Lett. 100, 247003 (2008).

${ }^{23}$ S. O. Valenzuela and V. Bekeris, Phys. Rev. Lett. 86, 504 (2001).

${ }^{24}$ S. O. Valenzuela, Phys. Rev. Lett. 88, 247003 (2002).

${ }^{25}$ N. Mangan, C. Reichhardt, and C. J. Olson Reichhardt, Phys. Rev. Lett. 100, 187002 (2008).

${ }^{26}$ A. B. Kolton, D. Domínguez, and N. Gronbech-Jensen, Phys. Rev. B 65, 184508 (2002).

${ }^{27}$ A. B. Kolton, D. Domínguez, and N. Gronbech-Jensen, Phys. Rev. Lett. 86, 4112 (2001).

${ }^{28}$ A. M. Campbell, J. Phys. C 4, 3186 (1971).

${ }^{29}$ M. W. Coffey and J. R. Clem, Phys. Rev. Lett. 67, 386 (1991).

${ }^{30}$ C. J. van der Beek, V. B. Geshkenbein, and V. M. Vinokur, Phys. Rev. B 48, 3393 (1993).

${ }^{31}$ E. H. Brandt, Phys. Rev. B 50, 4034 (1994). 